\title{
Diblock copolymer based self-assembled nanomagnetoelectric
}

\author{
Shenqiang Ren, Robert M. Briber, and Manfred Wuttig ${ }^{\text {a) }}$ \\ Department of Materials Science and Engineering, University of Maryland, College Park, \\ Maryland 20742, USA
}

(Received 28 August 2008; accepted 29 September 2008; published online 31 October 2008)

\begin{abstract}
A magnetoelectric (ME) composite with controlled nanostructures is synthesized using coassembly of two inorganic precursors with a block copolymer. This solution processed material consists of hexagonally arranged ferromagnetic cobalt ferrite $\left[\mathrm{CoFe}_{2} \mathrm{O}_{4}(\mathrm{CFO})\right]$ nano cylinders within a matrix of ferroelectric lead zirconium titanate $\left[\mathrm{Pb}_{1.1}\left(\mathrm{Zr}_{0.53} \mathrm{Ti}_{0.47}\right) \mathrm{O}_{3}(\mathrm{PZT})\right]$ when thin films were prepared by spin coating. The initial magnetic permeability of the self-assembled CFO/PZT nanocomposite changes by a factor of 5 through the application of $2.5 \mathrm{~V}$. This work also demonstrates that a block copolymer can be used to simultaneously template two compound inorganic phases to form a nanoscale composite. (C) 2008 American Institute of Physics. [DOI: 10.1063/1.3005558]
\end{abstract}

Nanostructured magnetoelectrics (MEs) have attracted much interest due to their potential applications. They may be divided into two categories, natural MEs such as $\mathrm{YbMn}_{2} \mathrm{O}_{5}$ and ME composites (MECs) such as eutectoidally decomposed solid solutions developed in the 1970s. ${ }^{1}$ The recent renaissance of MEs and MECs was spurred by the discovery of additional natural MEs (Ref. 2) and the expectation that large interaction coefficients could be achieved with MECs. ${ }^{3}$ Both self-organized and other types of MECs have since been fabricated, some with large interaction coefficients. ${ }^{4,5}$ The highest degree of self-organization has been achieved by three-dimensional epitaxial fabrication of one to three MECs (Ref. 6) and local electric field control of the magnetization on a microscale has recently been observed in a $\mathrm{Co}_{90} \mathrm{Fe}_{10} / \mathrm{BiFeO}_{3}$ heterostructure. ${ }^{7}$ In many of these structures the epitaxial elastic energies interfere with the magneto-and electromechanical contributions. Alternative routes to synthesize nanoscale self-organized MECs are therefore desirable.

Self-assembly of laminate MECs has been achieved by spinodal decomposition of a sol-gel precursor mixture in a magnetic field. ${ }^{8}$ MEC synthesis via self-assembly of diblock copolymers is very attractive as it can potentially yield a variety of composite morphologies with well-defined geometries. Furthermore, the block copolymer based selfassembly approach can provide simpler fabrication schemes.

Since the pioneering work of Kresge et al.,${ }^{10}$ numerous approaches have been developed using low or high molecular weight surfactants or amphiphilic block copolymers to direct the nanostructures of inorganics. Most of the work, directed toward mesoporous silica, used amphiphilic block copolymers or surfactants with sol-gel chemistry. ${ }^{11,12}$ Poly(ethylene oxide) (PEO) is widely used as the hydrophilic part of copolymers combining it with other hydrophobic polymers into diblock or triblock molecular architectures. Inorganic precursors other than silica have been also used for this coassembly approach: titania, ${ }^{13,14}$ niobium oxide, ${ }^{15}$ silicon carbonitrides, ${ }^{16}$ and silsesquioxanes. ${ }^{17,18}$ Many of these efforts have been reviewed recently. ${ }^{19}$ Two-phase inorganic composites have been synthesized by incorporating the com-

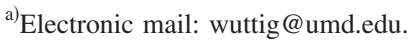

ponents into the interphase and one block of polystyrene-block-poly(ethylene-alt-propylene) ${ }^{20}$ None have shown the ability to simultaneously template two separate oxides in the blocks of a diblock copolymer, i.e., MECs, as will be demonstrated here.

For the synthesis of the desired MEC, $\mathrm{Pb}_{1.1}\left(\mathrm{Zr}_{0.53} \mathrm{Ti}_{0.47}\right) \mathrm{O}_{3}(\mathrm{PZT})$ and $\mathrm{CoFe}_{2} \mathrm{O}_{4}$ (CFO) components were dissolved in propylene glycol monomethyl ether and 2-methxyethanol, respectively, and combined with tetrahydrofuran (THF) which is a solvent for amphiphilic polystyrene-block-poly(ethylene oxide) (PS- $b$-PEO). An asymmetric PS- $b$-PEO $(65 / 35)$ was used to synthesize a hexagonally ordered MEC, more details in supplement section (SS) $1 .^{21}$ The block copolymer solution in THF $(10 \mathrm{mg} / \mathrm{ml})$ was mixed with the PZT and CFO precursor solutions with 1:1 ratio of inorganic precursor to PS or PEO solids, respectively. A thin film of block copolymer containing the sol-gel precursors was spin coated on a previously patterned silicon substrate. The films were subsequently exposed to chloroform vapor at room temperature for $24 \mathrm{~h}$ and afterward heated at a rate of $5{ }^{\circ} \mathrm{C} / \mathrm{min}$ to $500{ }^{\circ} \mathrm{C}$ where they were held for $2 \mathrm{~h}$.

Figure 1 shows atomic force microscopy (AFM) images of the thin films. Their surface displays hexagonally arrayed cylinders after solvent vapor annealing. The thermal treatment which induces crystallization of the inorganic precursors does not change the hexagonal-like order of the micro-
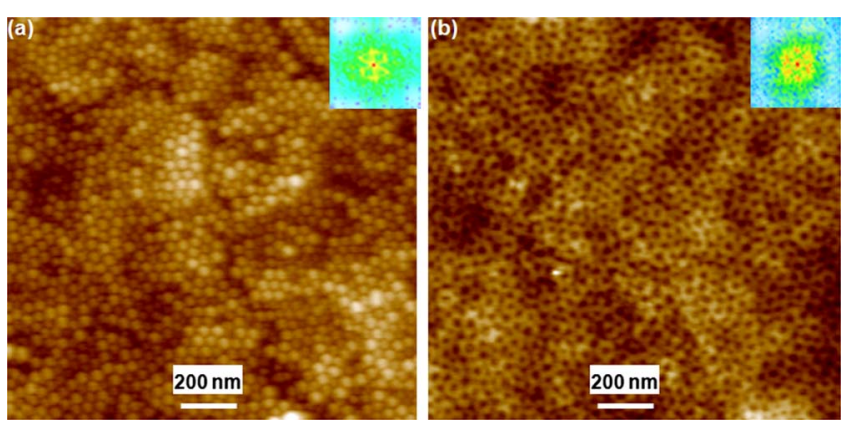

FIG. 1. (Color online) AFM images of the PS- $b$ PEO template before and after heat treatment; (a) after solvent annealing for $24 \mathrm{~h}$ at room temperature; (b) after heating at a rate of $5{ }^{\circ} \mathrm{C} / \mathrm{min}$ to $500{ }^{\circ} \mathrm{C}$ and holding for $2 \mathrm{~h}$. The (fast) Fourier transforms shown as (a) inserts confirm the hexagonal order of the self-assembled gel thin film, and (b) crystallized CFO cylinders. 


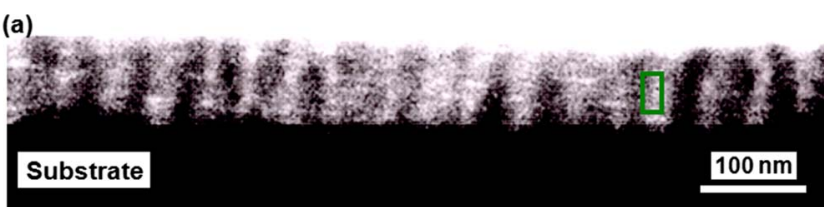

(b)
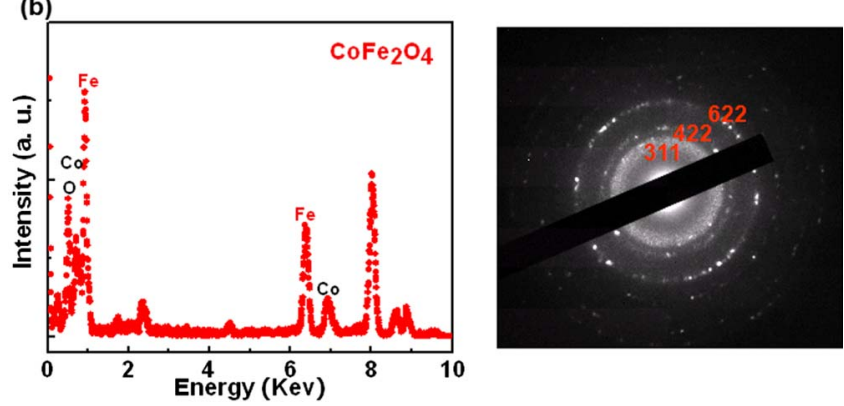

(c)
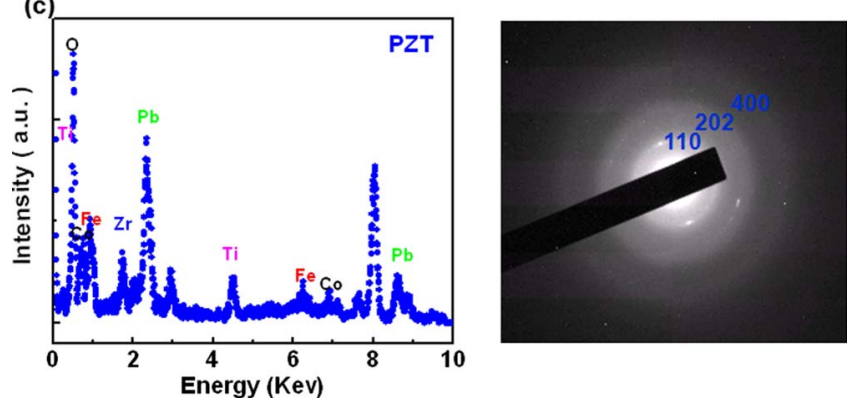

FIG. 2. (Color online) TEM results of self-assembled thin-film nanostructures; (a) cross sectional TEM image of the PZT/CFO nanocomposite and (b) microbeam EDS elemental composition analysis, as well as (c) results of microbeam characterization. The peak at $8 \mathrm{keV}$ originates from the $\mathrm{Cu}$ grid supporting the TEM specimen. Supplementary information is presented in Fig. S3.

domains as demonstrated by the insets of Fig. 1. The magnetic force microscopy image shown in SS $3^{21}$ indicates magnetic contrast and suggests the CFO columns are ferromagnetic. Their irregular magnetization is likely due to geometrical magnetic frustration. ${ }^{22}$

Further structural and analytical transmission electron microscopies (TEMs), including microbeam electron (beam size $\approx 10 \mathrm{~nm}$ ) diffraction and energy dispersive spectroscopy (EDS) (beam size $\approx 15 \mathrm{~nm}$ ) analyses, together with the AFM patterns, all shown in Figs. 2 and SS $5^{21}$, demonstrate collectively that the synthesized MEC consist of a hexagonal array of CFO nanocylinders that are embedded in a PZT matrix, as shown in SS $4^{21}$. The High-resolution TEM image of the interface marked in Fig. 2(a) and shown in SS 6, ${ }^{21}$ indicates that it is well sintered and, according to the orientation of the adjacent local grains, not epitaxial. Since the average MI response of the MEC can be accounted for using macroscopic CFO and PZT parameters and the dimensions of the MEC, the interface enables stress transfer from PZT to $\mathrm{CFO}$. The Fe profile across the CFO/PZT interface indicates only limited interdiffusion during the heat treatment.

SS $3^{21}$ indicates that the CFO cylinders are ferromagnetic. The ferroelectric nature of the PZT matrix is evident from Fig. 3(a). Figure 3(b) presents the cylinders' average magnetic and ME characteristics. The dipolar interaction between the hexagonally arranged CFO cylinders leads to an antiferromagnetic ground state with an almost zero remanence. The overall magnetic characteristics of the assembly can be understood in terms of the dipolat energy discussed in
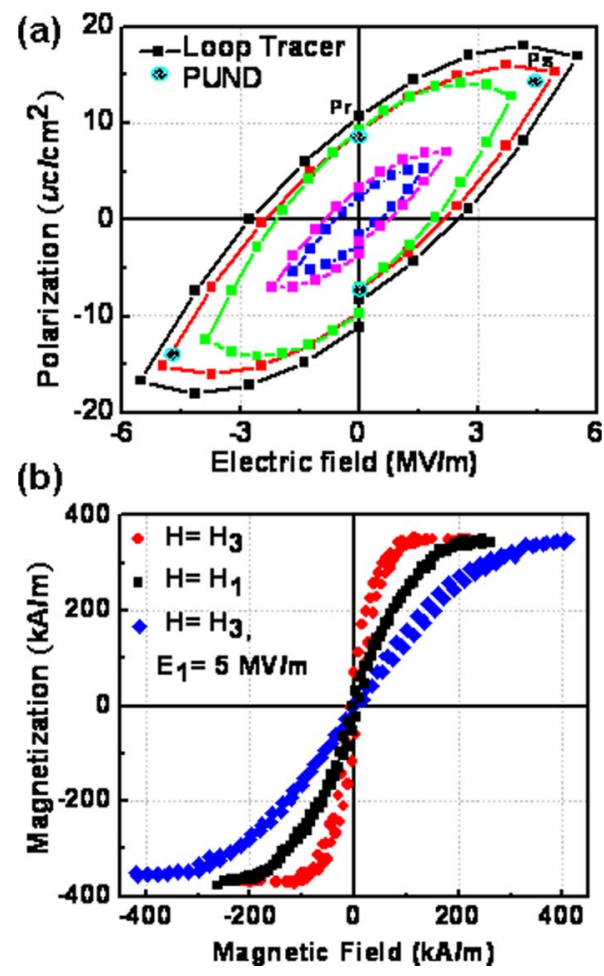

FIG. 3. (Color online) (a) Well-defined ferroelectric hysteresis of the annealed thin film; the saturation and remanent saturation polarization values, $P_{r}$ and $P_{s}$, resulted from polarization positive up-negative down measurements; (b) ferromagnetic and ME characteristics of the hexagonal CFO cylinder array embedded in a PZT matrix magnetized parallel and perpendicularly to their axes, $H_{3}(\bigcirc \bigcirc)$ and $H_{1}(\square \square)$, and with an applied magnetic, $H_{3}$ plus electric field, $E_{1}(\diamond \bullet)$. The permeability of the nanostructure can be changed by the application of an electric field. The ratio of the initial permeabilities, $\mu_{i}\left(H_{3}\right) / \mu_{i}\left(H_{3}, E_{1}\right) \approx 5$.

SS6, the demagnetization energy, see SS8, and the magnetomechanical anisotropy energy density of the CFO cylinders created by the polarized PZT matrix, $K_{E}$, derived in SS7. All are listed in Table I. The magnetocrystalline anisotropy energy has been omitted as the cylinders are nanopolycrystalline. Table I also contains numerical values for the theoretically and experimentally obtained numbers. Considering the approximations made, the agreement between the theoretically and experimentally determined anisotropies is satisfactory.

The present CFO/PZT nano-MEC corresponds to a one to three composite optimizing the ME interaction. ${ }^{23}$ Since the electro- and magnetomechanical interaction coefficients $d_{13}^{\text {PZT }}>0$ and $\lambda_{s}^{\mathrm{CFO}}<0$ an electric field, $E_{1}$, applied perpendicularly to the axis of the CFO cylinders, will reduce their average magnetization, as can be verified in Fig. 3(b). The total anisotropy energy of the cylinders magnetized parallel to their axes and subjected to a perpendicular electric field is given by $U_{\uparrow \uparrow}-U_{\uparrow \downarrow}+K_{E}$. Since all anisotropy energies are of similar magnitude, see SS8, an electric field applied to the PZT matrix will reduce the magnetization of the CFO cylinders noticeably, as confirmed by the data shown in Fig. 3(b). They demonstrate that the initial permeability of the CFO array, $\mu_{i}$, can be changed significantly by the electric field $E_{1}$ : the ratio $\mu_{i}\left(H_{3}\right) / \mu_{i}\left(H_{3}, E_{1}\right) \approx 5$ corresponding to a coupling coefficient $\mathrm{ME}_{E} \approx 0.05 \mathrm{~A} / \mathrm{V}$ equivalent to $\mathrm{ME}_{H}$ $\approx 10 \mathrm{~V} / \mathrm{A}$. Its magnitude is the result of the balanced magnetic, magneto- and electromechanical energies, the absence of epitaxial bias stresses and the rigidity of the interface. 
TABLE I. Theoretical and experimental values of the anisotropy energies. $U_{\uparrow \uparrow}$, interaction energy $U_{0}$ of parallel moments; $U_{\uparrow \downarrow}$, ditto of antiparallel moments; $U_{\rightarrow \rightarrow}$, ditto of collinear moments; $\Delta U_{d}$, change in demagnetization energy; $\lambda_{s}$, saturation magnetostriction of $\mathrm{CFO} ; d_{13}$, piezoelectric coefficient of PZT; $M_{S}$, saturation magnetization of CFO cylinders; $K_{E}$, ME anisotropy energy; $U_{0}$, value of the dipolar interaction energy; $(Y)$ average elastic constant of PZT/CFO, see SS7; and $V_{c}$, volume of cylinder.

\begin{tabular}{lllcc}
\hline \hline & & \multicolumn{2}{c}{ Theory } & \\
\cline { 2 - 4 } Transition & Anisotropy Energy & Expression per cylinder & Value & $\begin{array}{c}\text { Expt. } \\
(\mathrm{eV})\end{array}$ \\
\hline $\begin{array}{l}\text { Axial antiferro } \rightarrow \\
\text { axial ferro }\end{array}$ & $U_{\uparrow \uparrow}-U_{\uparrow \downarrow}$ & $\frac{1}{2} U_{0}$ & 6 & 7 \\
$\begin{array}{l}\text { Axial antiferro } \rightarrow \\
\text { radial ferro }\end{array}$ & $U_{\uparrow \uparrow}-U_{\uparrow \downarrow}+\Delta U_{d}$ & $\frac{1}{2} U_{0}+\frac{1}{4} \mu_{0} M_{S}^{2} V_{c}$ & 17 & 16 \\
$\begin{array}{l}\text { Axial antiferro } \rightarrow \\
\text { axial ferro }+E_{1}\end{array}$ & $U_{\uparrow \uparrow}-U_{\uparrow \downarrow}+K_{E}$ & $\frac{1}{2} U_{0}+\lambda_{s} d_{13} E_{1}\langle Y\rangle V_{c}$ & 34 & 27 \\
\hline \hline
\end{tabular}

In conclusion, a, simple and inexpensive synthesis route to highly dense arrays of self-organized patterned MECs using thin-film block copolymers combined with sol-gel chemistry was developed and used to fabricate a strongly coupled MEC. This work demonstrated templating of two compound inorganic materials to form a nanoscale composite using a block copolymer. Thin films with a CFO precursor in a cylindrical PEO domain and a PZT precursor in the surrounding PS matrix were obtained by spin coating and subsequent heat treatments lead to degradation of the organic material and crystallization of the inorganic phases. The experimental results suggest that the PZT and CFO precursors were selectively incorporated into the PS and PEO blocks, respectively, permitting the formation of the PZT and CFO phases largely independently while maintaining the structure templated by the block copolymer. The method is applicable to the synthesis of any two-phase inorganic composite. Specific material combinations and patterns can be selected through the nature of the precursors and the ratio of the diblock copolymer. The ferromagnetic, ferroelectric, and ME characteristics are equally tunable over a wide range of parameters as can be seen from the expressions for the controlling magneto- and electromechanical energy densities given in the supplement.

The comments of Dr. Ho-Cheol Kim are gratefully acknowledged. This work was supported by the Grant and Contract Nos. NSF-DMR0705368, ONR-MURI N000140110761, and ARO-MURI 28D1083899.

${ }^{1}$ J. Van den Boomgaard, A. Van Run, and J. Van Suchtelen, Ferroelectrics 10, 295 (1976)

${ }^{2}$ S. W. Cheong and M. Mostovoy, Nature Mater. 6, 13 (2007).
${ }^{3}$ C. W. Nan, M. I. Bichurin, S. Dong, and D. Viehland, J. Appl. Phys. 103, 031101 (2008).

${ }^{4}$ R. Ramesh and N. A. Spaldin, Nature Mater. 6, 21 (2007).

${ }^{5}$ W. Eerenstein, N. D. Mathur, and J. F. Scott, Nature (London) 442, 759 (2006).

${ }^{6} \mathrm{H}$. Zheng, Nature (London) 303, 661 (2004).

${ }^{7}$ Y. Chu, L. W. Martin, M. B. Holcomb, M. Gajek, S. Han, Q. He, N. Balke, C. Yang, D. Lee, W. Hu, Q. Zhan, P. Yang, A. Fraile-Rodriguez, A. Scholl, S. X. Wang, and R. Ramesh, Nature Mater. 7, 478 (2008).

${ }^{8}$ S. Ren and M. Wuttig, Appl. Phys. Lett. 91, 083501 (2007).

${ }^{9}$ P. Judeinstein and C. J. Sanchez, J. Mater. Chem. 6, 511 (1996).

${ }^{10}$ C. T. Kresge, M. E. Leonowicz, W. J. Roth, J. C. Vartuli, and J. S. Beck, Nature (London) 359, 710 (1992).

${ }^{11}$ M. Templin, A. Frank, A. DuChesne, H. Leist, Y. M. Zhang, R. Ulrich, V. Schadler, and U. Wiesner, Science 278, 1795 (1997).

${ }^{12}$ Q. Huo, R. Leon, P. M. Petroff, and G. D. Stucky, Science 268, 1324 (1995).

${ }^{13}$ S. Haseloh, S. Y. Choi, M. Mamak, N. Coombs, S. Petrov, N. Chopra, and G. A. Ozin, Chem. Commun. (Cambridge) 2004, 1460.

${ }^{14}$ P. C. A. Alberius, K. L. Frindell, R. C. Hayward, E. J. Kramer, G. D. Stucky, and B. F. Chmelka, Chem. Mater. 14, 3284 (2002).

${ }^{15}$ J. Lee, M. C. Orilall, S. C. Warren, M. Kamperman, F. J. Disalvo, and U. Wiesner, Nature Mater. 7, 222 (2008).

${ }^{16}$ M. Kamperman, C. B. W. Garcia, P. Du, H. Ow, and U. Wiesner, J. Am. Chem. Soc. 126, 14708 (2004).

${ }^{17}$ E. M. Freer, L. E. Krupp, W. D. Hinsberg, P. M. Rice, J. L. Hedrick, J. N. Cha, R. D. Miller, and H. C. Kim, Nano Lett. 5, 2014 (2005).

${ }^{18}$ L. Sundström, L. E. Krupp, E. Delenia, C. Rettner, M. Sanchez, M. Hart, Y. Zhang, and H. C. Kim, Appl. Phys. Lett. 88, 243107 (2006).

${ }^{19}$ S. B. Darling, Prog. Polym. Sci. 32, 1152 (2007).

${ }^{20}$ M. Bockstaller, Y. Lapetnikov, S. Margel, and E. L. Thomas, J. Am. Chem. Soc. 125, 5276 (2003).

${ }^{21}$ See EPAPS Document No. E-APPLAB-93-011843 for synthesis, characterization and calculation of the PZT/CFO Composite. For more information on EPAPS, see http://www.aip.org/pubservs/epaps.html

${ }^{22}$ A. P. Ramirez, in Handbook of Magnetic Materials (North-Holland, Amsterdam, 2001), Vol. 13, Chap. 4.

${ }^{23}$ R. Ramesh, Nat. Nanotechnol. 3, 7 (2008). 